\title{
EPISTEMOLOGÍA GENÉTICA Y LA (IN)VISIBILIDAD \\ DE LOS ENFOQUES CONSTRUCTIVISTAS EN NEUROCIENCIAS
}

EPISTEMOLOGIA GENÉTICA E A (IN)VISIBILIDADE

DAS ABORDAGENS CONSTRUTIVISTAS NAS NEUROCIÊNCIAS

Genetic epistemology and the (in)visibility

of constructivist approaches in neurosciences

\author{
TAís Oliveira Martins ${ }^{*}$ \\ Universidade Federal do Rio Grande do Sul (UFRGS), Porto Alegre (RS) / Brasil \\ oliveiramartins.tais@gmail.com \\ Código Orcid: https://orcid.org/0000-0003-2198-0186 \\ MARCELO LEANDRO EICHLER \\ Universidade Federal do Rio Grande do Sul (UFRGS), Porto Alegre (RS) / Brasil \\ marcelo.eichler@ufrgs.br \\ Código Orcid: http://orcid.org/0000-000 1-5650-9218
}

Forma sugerida de citar: Oliveira, Taís \& Eichler, Marcelo (2019). Epistemología genética y la (in)visibilidad de los enfoques constructivistas en neurociencias. Sophia, colección de Filosofía de la Educación, 26(1), pp. 115-140.

* Tiene una licenciatura en Química (2009) por la Universidad Luterana do Brasil (Ulbra), especialización en los medios de comunicación en la Educación (2014) por el Instituto Federal de Educación, Ciencia y Tecnología en África (IFSul Riograndense) y maestría en Educación de la Universidade Federal do Rio Grande do Sul (UFRGS).

** Tiene una licenciatura en Química (1997), maestría en Psicología (2000) y doctorado en Psicología del desarrollo (2004), obteniendo todos los títulos de la Universidad Federal de Rio Grande do Sul. Actualmente es profesor adjunto en el Departamento de Química Inorgánica, Universidad Federal de Rio Grande do Sul (UFRGS) y profesor titular del programa de posgrado en educación (UFRGS). 


\section{Resumo}

Este trabalho discute o atual debate sobre o campo das neurociências cognitivas, sua possível relação com a epistemologia genética de Jean Piaget e os motivos pelos quais as abordagens construtivistas nas neurociências têm sido pouco promovidas. Para demonstrar que as neurociências não constituem uma área singular, unânime e finalizada como institui o senso comum, propomos uma revisão teórica dos estágios de desenvolvimento de Piaget e a discussão do paralelismo psicofisiológico defendido por ele. Assim, apresentamos evidências neurobiológicas apontadas por Herman T. Epstein para a ocorrência de Frenoblenoses (períodos de rápido crescimento cerebral), intercalados com períodos de crescimento lento e que ocorrem em correlação com os estágios piagetianos. Com isto, busca-se traçar aproximações possíveis entre a epistemologia genética de Piaget e as neurociências cognitivas, demonstrando que o desenvolvimento cognitivo humano está estruturado nos pilares psicológico e biológico, e que estes estão ligados a fatores sociais, culturais, educativos e genéticos; além de demonstrar que a base de sustentação do pólo construtivista nas neurociências é bastante sólida. Discutimos, também, que a análise superficial das evidências ou dos modelos piagetianos, a adoção de parcialidade e a omissão de dados, a não consideração dos trabalhos dele como um todo, o apego a obras isoladas em determinados períodos, leituras secundárias e terciárias da obra piagetiana e os problemas de leitura e tradução dos textos originais impedem a visão de Piaget como epistemólogo e justificam a pouca divulgação da abordagem construtivista nas pesquisas neurocientíficas.

\section{Palavras-chave}

Cérebro, desenvolvimento mental, psicologia do desenvolvimento, epistemologia, neurobiologia

\section{Resumen}

Este artículo discute el actual debate en el área de las neurociencias cognitivas, su posible relación con la epistemología genética de Jean Piaget y los motivos por los cuales los enfoques constructivistas en las neurociencias han sido poco utilizadas. Para demostrar que las neurociencias no constituyen un área singular, unánime y finalizada como instituye el sentido común ilustrado, se presenta una revisión teórica de los estadios de desarrollo de Piaget y la discusión del paralelismo psico-fisiológico defendido por él. A continuación, se presentan evidencias neurobiológicas apuntadas por Herman T. Epstein para la ocurrencia de Frenoblenosis (períodos de rápido crecimiento cerebral), intercalados con períodos de crecimiento lento, que estarían relacionados a las etapas piagetianas. De esta forma, se busca trazar aproximaciones posibles entre la epistemología genética de Piaget y las neurociencias cognitivas, demostrando que el desarrollo cognitivo humano está estructurado en los pilares psicológico y biológico, y que éstos están ligados a factores sociales, culturales, educativos y genéticos; además de demostrar que la base de sustentación del polo constructivista en las neurociencias es bastante sólida. Se discute, también, algunos posibles motivos para la negligencia de la obra de Piaget en el área de las neurociencias, donde se apunta: el análisis superficial de las evidencias o de los modelos piagetianos, la no consideración de los trabajos de él como un todo, el apego a obras aisladas en determinados períodos, lecturas secundarias y terciarias de la obra, entre otros. Tales equívocos de lectura impedirían la visión de Piaget como epistemólogo y justifican la poca divulgación del abordaje constructivista en las investigaciones neurocientíficas.

\section{Palabras clave}

Cerebro, desarrollo mental, psicología del desarrollo, epistemología, neurobiología.

\section{Abstract}

This paper discusses the current debate about the field of cognitive neurosciences, its possible relation with the genetic epistemology of Jean Piaget and the reasons why the constructivist approaches in the neurosciences have been little promoted. In order to demonstrate that the 
neurosciences don't constitute a singular, unanimous and finalized area as it establishes the common sense, is proposed a theoretical revision of the stages of development of Piaget and the discussion of the psycho-physiological parallelism defended by him. Thus, is presented the neurobiological evidences pointed out by Herman T. Epstein for the occurrence of Phrenoblysis (periods of fast brain growth), interspersed with periods of slow growth and that occurring in correlation with the Piagetian stages. With this, we search to draw possible approximations between the genetic epistemology of Piaget and cognitive neurosciences, demonstrating that human cognitive development is structured in the psychological and biological pillars, and that these are linked to social, cultural, educational and genetic factors as well as to demonstrate that the base of support of the constructivist pole in the neurosciences is quite solid. Also is discussed that the superficial analysis of Piagetian precepts, the adoption of partiality and omission of data, the failure to consider his works as a whole, the attachment to isolated works in certain periods, secondary and tertiary readings of the Piagetian work and the problems of reading and translation of the original texts impede Piaget's view as epistemologist and justify the little dissemination of the constructivist approach in neuroscientific research.

\author{
Keywords \\ Brain, mental development, development psychology, epistemology, neurobiology
}

\title{
Introducción
}

El presente artículo pretende discutir el actual debate que envuelve el campo de las neurociencias cognitivas, su posible relación con la epistemología genética de Jean Piaget (1896-1980) y los motivos por los cuales los enfoques constructivistas en las neurociencias han sido poco promovidos. Serán presentadas en el transcurso del texto, que está estructurado en tres sesiones, evidencias de la polarización de este debate, principalmente en lo que se refiere a los enfoques que apuntan a la validez de la teoría piegetiana, con el fin de demostrar que las neurociencias no constituyen un área singular, unánime y finalizada como establece el sentido común ilustrado. Al contrario, el debate sobre el constructivismo en las neurociencias permanece abierto.

Por lo tanto, la primera sesión aborda la cuestión del desarrollo cognitivo para Piaget, donde se presentan las fases de desarrollo y las críticas puestas a este tema. En seguida, en la segunda sesión, se presenta la discusión sobre el paralelismo psico-fisiológico de Piaget, en el que se discuten las interacciones entre los factores biológicos y el medio físico en la constitución del sistema epigenético. Al final, la última sesión aborda las etapas cerebrales de Herman T. Epstein (1920-2007), ocasión en que se muestra que sus estudios sobre el desarrollo cerebral están directamente relacionados a la teoría piagetiana.

Aunque las neurociencias solo hayan adquirido su actual status recientemente - los grandes avances al final del siglo XX le dieron a su 
última década el título de 'Década del Cerebro'- durante el camino recorrido el impacto de creencias enfocadas hacia la ausencia de influencias del medio y para la finalización del sujeto centrado en su herencia genética/cromosómica dejó marcas profundas en el conocimiento difundido sobre este tema. Aún es posible encontrar incredulidad en la unión entre las neurociencias cognitivas y la epistemología genética, como si de forma automática una anulase la otra.

Becker (2003) presenta que el gran desafío del aprendizaje humano está en la superación de las concepciones fundamentadas en epistemologías del sentido común, sean ellas innatistas o empíricas. Machado (2015) comenta sobre la dicotomía entre el sentido común y el conocimiento científico y el hecho de que ese sentido común tiende a no aceptar al ser humano como 'marcadamente orgánico', mientras las neurociencias y sus cono-

118 cimientos científicos traen un entendimiento sobre el organismo humano enfatizando el contexto biológico. En ese contexto, se pode atentar a lo que Corso (2009) presenta sobre plasticidad cerebral, mostrándola como muy reciente y destacando que hasta entonces las neurociencias presentaban el sistema nervioso como una estructura programada, no modificable.

Según Machado (2015) se hace necesaria la desvinculación con el sentido común que tiene dificultades en aceptar ese carácter 'marcadamente orgánico' de los humanos y también con las escuelas académicas que intentan mostrar la humanidad como únicamente biológica. La comprensión del sujeto epistemológico y del estudio realizado para esa comprensión necesita del carácter dual (enfoques psicológico y fisiológico integrados) como presentado por Piaget y defendido en el área neurocientífica, por ejemplo, por Herman T. Epstein, Steven R. Quartz, Terrence Sejnowski, William J. Hudspeth, Karl H. Pribram y António Damásio. Sin embargo, es importante atender al echo destacado por Corso (2009) de que Piaget "consideró la maduración del sistema nervioso una condición necesaria, aunque no suficiente, del desarrollo cognitivo" (p. 229).

Muchas visiones y muchos enfoques son dados cuando el asunto son las neurociencias. Muchas veces, ni siquiera es utilizado el término en plural, denotando una ciencia singular, única, estandarizada, unánime. Correa, Agila, Pulamarín y Palacios (2012) presentan como expresión común llamar de 'neuromitos' a las interpretaciones equivocadas sobre los descubrimientos del campo neurocientífico, lo que demuestra cierta frecuencia de estos acontecimientos. Incluso en áreas aplicadas, como la neuroeducación, donde se discute un campo transdisciplinar (educación, psicología, neurociencias, etc.), se presenta una visión finalista y estandarizada. 
Un ejemplo de eso ocurre cuando Aranha y Sholl-Franco (2012) muestran el campo de la neuroeducación como responsable de presentar "métodos y técnicas que optimicen, o incluso posibiliten en algunos casos, una mejor relación profesor-alumno y enseño-aprendizaje" (p. 11). Solo pensar la posibilidad de que todo un campo científico, transdisciplinar y heterogéneo pueda tener recetas de éxito listas a ser aplicadas ya denota un posicionamiento por la singularización y estandarización de este campo.

Otro ejemplo a abordar es la investigación de Hansen y Monk (2002), en que desarrollaron un trabajo que se constituyó en una revisión de búsquedas sobre desarrollo cerebral y estructuras de aprendizaje. Ellos construyeron propuestas para el análisis del aprendizaje en educación en ciencias, que se volvió una de las pocas referencias de este tema dentro del enseño científico y de la didáctica de las ciencias.

Utilizando técnicas de imagen de Electroencefalograma (EEG), Potencial Cerebral en Eventos Relacionados (EEG/ERP) y Resonancia Magnética (MRI) para acompañar los mecanismos de funcionamiento cerebral mientras el sujeto realizaba las tareas propuestas, el trabajo de esos autores proporcionó evidencias de que la maduración cerebral puede estructurar la plasticidad disponible para la construcción de la mente, y permitió la proposición de las implicaciones de este conocimiento para la educación en ciencias. Sin embargo, al realizar la discusión de su estudio, ellos atraen investigadores con visiones diferentes (Alexander Romanovich Luria (1902-1977), Herman T. Epstein (1920-2007), Jean-Pierre Changeux y Stanislas Dehaene) deseando discutir la madures del córtex pre-frontal, ellos consiguieron demostrar la neuroplasticidad siendo más larga en el cerebro en madures de lo mostrado hasta aquel momento y, también, que el cerebro humano inicialmente tiene un exceso de conexiones sinápticas y realiza podas sosteniendo algunas conexiones mientras otras son removidas. Sin embargo, el no abordaje de las diferentes visiones utilizadas por los investigadores citados en el estudio acabó una vez más reforzando la idea de que las neurociencias tienen una comprensión homogénea y no conflictiva de su campo de estudio.

El conflicto interno de esa área está básicamente polarizado entre un campo innatista y otro constructivista. Eichler y Fagundes (2005), al discutir el célebre debate entre Piaget y Chomsky, traen este análisis para el foco y demuestran los puntos de vista y enfoques dados por ambos polos del debate. Utilizando un sesgo piagetiano en la lectura de la neurobiología, los autores critican la visión innatista de Chomsky y de Changeux, aceptada y defendida por muchos, y demuestran que existi- 
rían evidencias a sugerir, que la discusión sobre el constructivismo en las neurociencias aún está en abierto.

En ese sentido, se puede citar el manifiesto constructivista de Quartz y Sejnowski (1997), que propuso presentar la base neural para el desarrollo cognitivo, llamado por ellos de constructivismo neuronal, como un ejemplo del polo constructivista de este debate. Por medio de este manifiesto, ellos divulgaron evidencias neurobiológicas de que las características representacionales del córtex cerebral son construidas en la interacción dinámica entre los mecanismos de crecimiento neural y las actividades neuronales derivadas del ambiente, cuyo crecimiento está siendo mostrado como un aumento progresivo de las propiedades representacionales del córtex. No se debe ver la evolución cognitiva humana simplemente como el aumento de estructuras especializadas. El cons120 tructivismo neuronal muestra, por el contrario, que es el incremento de la flexibilidad de las representaciones que permite a los factores ambientales moldar la estructura y el funcionamiento del cerebro humano.

Se ha discutido que el campo neurocientífico es vasto, no homogéneo, polarizado en el debate innatismo $\mathrm{x}$ constructivismo, $\mathrm{y}$ así se ve que el sentido común ilustrado del tema no ha tomado en cuenta estas discusiones. A pesar de las corrientes que afirman la invalidad de la teoría constructivista, es posible notar entre ellas un comportamiento bastante específico: la supuesta invalidad de la misma viene en medio de un estudio superficial de sus modelos, de la adopción de una parcialidad que lleva a la omisión de datos, la no consideración de los trabajos de Piaget como un todo, el apego a obras aisladas en determinados períodos, lecturas secundarias y terciarias de la obra piagetiana, problemas de lectura y traducción de los textos originales, entre otros problemas de lectura, como apuntan, por ejemplo, Eichler y Fagundes (2005). La discusión de Lourenço (2016) sobre la 'visión recibida' de la obra de Piaget deja estos puntos bien aclarados.

Un ejemplo de estudio superficial de la obra piagetiana es resaltado por Dongo-Montoya al hablar sobre las críticas hechas por Lev Vygotsky (1896-1934) al trabajo de Piaget. Al analizar cuestiones levantadas para criticar el egocentrismo lingüístico, Dongo-Montoya (2013) afirma:

A continuación, Piaget afirma que sus críticos se detuvieron en la observación del primer capítulo de su obra El Lenguaje y el Pensamiento del Infante (1999). En ese capítulo, Piaget coloca como manifiesto un inventario del habla espontánea del infante, tratando de distinguir los monólogos individuales y los monólogos colectivos de las comunicaciones adaptativas (cooperativas). En el segundo y en el tercer capítulo, se estudian las conversaciones y los argumentos de los infantes, los cuales 
tienen por objeto superar sus propios puntos de vista. Sin embargo, esos estudios no fueron tomados en consideración por la crítica (p. 278).

Otro buen ejemplo, ahora demostrando la adopción de parcialidad y omisión de datos, viene de la posición adoptada por Maximo PiattelliPalmarini, editor del libro con el debate entre Piaget y Chomsky. Para Eichler y Fagundes (2005), el autor demuestra:

[...] tendencia a desautorizar, silenciar y/o eliminar la perspectiva que es divergente en relación a la que adopta, usando con habilidad, el arsenal retórico es bastante utilizado por Piattelli-Palmarini y puede ser evidenciado en las introducciones y en las conclusiones que hace a cada capítulo del libro (p. 258)

Según Eichler y Fagundes (2005), "la defensa del programa científico de Chomsky es hecha, algunos años más tarde, por el organizador del libro originado" (p. 256). Sin embargo, el investigador comprometido hará exactamente lo contrario, y en su búsqueda por el todo irá a deconstruir los estereotipos y descubrirá que muchos datos actuales y nuevas búsquedas corroboran el trabajo piagetiano, como las actuales investigaciones neurobiológicas que muestran es al menos precipitado sostener el crédito de las contribuciones constructivistas. El debate, por lo tanto, está aún en abierto y de momento ha creado más polarización que búsqueda productiva.

De esta forma, este artículo se suma en sentido de mantener este debate encendido. Para esto, se presenta una revisión que parece ir contra el sentido común ilustrado y el discurso que promueve el campo neurocientífico idealizado, homogéneo, infalible, finalista y, en fin, singular. Presentando algunas ideas sobre los enfoques constructivistas en las neurociencias, que han sido poco difundidas, se especula sobre los motivos de los borrados y silencios en relación a las controversias epistemológicas en las neurociencias. En fin, se sugiere que, si nada cambia, la sociedad continuará creyendo que existe solo una neurociencia, y que ella es la poseedora de una sabiduría singular, unánime y terminada.

\section{El desarrollo cognitivo para Piaget}

Sin duda, las etapas del desarrollo cognitivo propuestas por Piaget han sido blanco de grande parte de las críticas envueltas en el debate que aquí se discute. Puntos de vista opuestos y muchas proposiciones intentan analizar el contexto, el método y el enfoque piagetianos (ej. Houdé, 2009; Niaz, 1998; Lourenço, 2016). Houdé (2009) apunta las fases del desarro- 
llo piagetianos como ocurriendo de forma lineal y acumulativa. Carey, Zaitchik y Bascandziev (2015), afirmaron que el trabajo de Piaget "tiene dos teorías esenciales: constructivismo y la teoría de las fases” (p. 36). Siguiendo otro sesgo, Lourenço (2016) apunta que las etapas del desarrollo han sido "centro de considerable investigación empírica y controversia teórica" (p. 123), y discute sobre una "visión recibida" de la teoría de Piaget que, aliada a una débil comprensión acerca del desarrollo, son los responsables por los malos entendidos al respecto del tema. Subía y Gordón (2014) afirman que en los orígenes de la psicología no había la presencia de estructuras cognitivas y que fue Piaget quien estableció una progresión en las etapas. Niaz (1998) destacó varios investigadores que rechazaron la teoría de Piaget por considerar el desarrollo en etapas muy heterogéneas, él enseguida apunta que los críticos interpretaron mal el 122 significado de las etapas de desarrollo intelectual humano.

Ampliamente divulgada, la teoría piagetiana ha sido abordada en libros didácticos y literatura enfocada a los campos de la psicología de desarrollo y de la pedagogía (educación en general). Muchos de estos libros didácticos (ej. Boyd y Bee, 2011; Bee y Boyd, 2011, Papalia y Feldman, 2013; Gazzaniga y Heatherton, 2005), presentan ideas extremamente cortas, conteniendo apenas puntos-clave y muchas veces reforzando la idea de que la teoría piagetiana gira en torno de las etapas de desarrollo cognitivo. Boyd y Bee (2011), por ejemplo, utilizan una página para presentar la teoría de Piaget y las etapas de desarrollo cognitivo son presentadas únicamente en un cuadro que ocupa casi la mitad de una página.

$\mathrm{Al}$ abordar el tema de las etapas del desarrollo cognitivo de Piaget, Lourenço (2016) destaca una serie de puntos fundamentales: (1) la visión de Piaget como un epistemólogo y no psicólogo, y su interés enfocado en el surgimiento de nuevas formas de conocimiento y no en el desarrollo individual; (2) se destaca el echo de Piaget nunca haber abandonado las etapas del desarrollo, y de que eso "no implica en que las etapas estén en el centro de su teoría" (p. 124); (3) la reafirmación de la creencia de Piaget de que el desarrollo nunca acaba, y de que las "estructuras formales pueden ser infinitamente elaboradas, extendidas, diferenciadas, compuestas y transformadas" (p. 128); (4) los factores debido a los cuales se deben los cambios de desarrollo apuntados por Piaget son la madurez biológica, la experiencia física y la interacción social (donde fue incluido el lenguaje); (4) las críticas a la teoría piagetiana son apuntadas como resultantes de lecturas hechas a partir de traducciones pobres, de la no lectura de textos originales (por no haber sido traducidos del original en francés, y por tanto de la falta de comprensión de Piaget como un epistemólogo); y por fin, (5) el apuntamiento 
de la importancia de las etapas del desarrollo para los estudios realizados por la psicología del desarrollo y su afirmación reiterada de que "si las etapas no existiesen, nosotros deberíamos inventarlas" (p. 132).

En relación a la periodización del desarrollo cognitivo, Piaget (1956) dividió el desarrollo intelectual en tres grandes períodos: el período de la inteligencia sensorio-motora, el período de preparación y organización de las operaciones concretas, y el período de las operaciones formales. El abordaje de las características apuntadas como las principales descriptoras de estos períodos, se apresta a seguir con un carácter exploratorio y no profundo, con la intención de fundamentar la discusión que proponemos adelante.

Sobre el período de la inteligencia sensorio-motora, resumidamente, se puede apuntar que Piaget $(1956,1983)$ presentó el recién-nacido como no, siendo titular de una consciencia de su yo, ni de los límites de su interior y exterior. Este período está marcado por la indiferenciación del sujeto en relación al mundo y a los otros, y por la centración de ese sujeto en sí mismo. Cerca de un mes después del nacimiento, el bebé pasa a demostrar los primeros hábitos, llamados condicionamientos estables y reacciones circulares primarias, que son relativas al propio cuerpo, como, por ejemplo, chupar el dedo o las manos. Alrededor de los 4 meses y medio se inicia la coordinación de la visión y de la prensión y el inicio de las reacciones circulares secundarias, que son dirigidas a los cuerpos manipulados (como mirar, coger, etc.). Sin embargo, el bebé aún no busca por un objeto desaparecido ya que su referencia continúa siendo el propio cuerpo pues la fijación, siendo inconsciente, permanece.

La construcción del Yo solo es posible en la medida en que el sujeto pasa a interesarse por el otro y la permanencia de los objetos se muestra primeramente cuando o lactante busca por la figura de ese otro (la madre, o cuidador, etc.). Alrededor de los 8 meses se inicia la coordinación de esquemas secundarios, donde el bebé pasa a utilizar medios conocidos para alcanzar nuevos objetivos y es cuando se inicia la búsqueda por el objeto desaparecido. Con cerca de 11 meses inicia la diferenciación de los esquemas de acción por reacción circular terciaria y el descubrimiento de medios nuevos, como tirar de una manta para sí o buscar el objeto desaparecido en diferentes lugares cuando estos desplazamientos sucesivos son hechos de forma perceptible. Al fin, en torno a los 18 meses tiene inicio la interiorización de los esquemas y soluciones de algunos problemas con la prohibición de la acción y la comprensión brusca. Según Piaget (1983), la revolución que se establece en el período sensorio-motor consiste en: 
...descentralizar las acciones en relación al cuerpo propio, en considerar este como objeto entre los demás en un espacio que a todos contiene y en asociar las acciones de los objetos bajo el efecto de las coordinaciones de un sujeto que comienza a conocerse como fuente o incluso señor de sus movimientos (p. 8).

Después de este período, se sigue un próximo que es el de preparación y organización de las operaciones concretas. Piaget (1956) llamó este subperíodo de 'representaciones pre-operatorias'. Ese período tendría inicio alrededor de 2 años y se extendería hasta cerca de 7-8 años. Alrededor de los 2 años, se manifiesta el surgimiento de la función simbólica e inicio de la interiorización de los esquemas de acción en representaciones, que aparecen de diferentes formas en el lenguaje, juego simbólico o imaginación, imitación. Alrededor de los 4 años se inician las organizaciones representativas basadas en las configuraciones estáticas, o en una asimilación a la propia acción, caracterizadas por interrogaciones sobre objetos para manipular y no conservación de los conjuntos y de las cantidades. Sobre los cambios observados, Piaget (1983) afirma:

...el sujeto se vuelve rápidamente capaz de inferencias elementales, de clasificaciones en configuraciones espaciales, de correspondencias, etc. (...) a partir del aparecimiento precoz de los "por qué?" estamos viendo un inicio de explicaciones causales (p.12).

Según Piaget (1983), el gran cambio en relación al período sensorio-motor se debe a la capacidad de conceptualización, ya que hasta entonces no hay una toma de consciencia y apenas la utilización material y práctica de los esquemas de inteligencia. Sin embargo, el también refuerza que ese pasaje de acción al pensamiento, o del esquema sensorio-motor al concepto es fruto "de una diferenciación lenta y laboriosa, que se relaciona a las transformaciones de la asimilación" (p. 13). Entre los 5 y 7-8 años se espera una fase intermedia entre la no conservación y la conservación, donde el niño inicia los enlaces entre estados y transformaciones, marcada por el inicio de una descentración entre conceptos o acciones conceptualizadas, diferentemente del periodo sensorio-motor cuando esa descentración se daba en relación a los movimientos, inicialmente ligados al propio cuerpo (de forma inconsciente). La descentración, vista en este período, permite al establecimiento de las funciones constituyentes (aún no constituidas), pues aún no son cuantitativas, sino cualitativas u ordinales. Además de eso, esa función constituyente no es reversible y, por lo tanto, no comporta las conservaciones que son características de 
las operaciones. Por lo tanto, no hay aún conservación de conjuntos o de cantidades de materia.

Enseguida, iniciando alrededor de los 7-8 años y extendiéndose hasta cerca de 11-12 años, se pasa al 'sub periodo de las operaciones concretas'. Según Piaget (1956) es donde se observan diferenciaciones progresivas, coordinaciones graduales, que llevan a las primeras manifestaciones de reversibilidad y conservación. Durante el desarrollo de este período, así como en los anteriores, los sujetos se desenvuelven gradualmente, de forma lenta y no abrupta, y pasan a demostrar niveles más complejos de inteligencia. La forma como el sujeto pasa a resolver las situaciones-problema a las que es sometido se caracterizan por la elaboración de soluciones que parten de informaciones o dados parciales, caracterizando las clasificaciones operatorias, reversibilidad operatoria, causalidad operatoria, etc. Explicando estas cuestiones, Piaget (1983) apunta que:

...las operaciones "concretas" recaen directamente sobre los objetos: esto equivale, pues, todavía a actuar sobre ellos, como en los niveles pre-operatorios, pero dando a esas acciones (o a aquellas que les son atribuidas cuando son consideradas como operaciones causales) una estructura operatoria, esto es, componible de manera transitiva y reversible (p. 23).

Alrededor de los 9 años, un segundo nivel de las 'operaciones concretas' comienza a establecerse, donde es golpeado el equilibrio general de estas operaciones principalmente en lo que habla respecto a la causalidad. Además, nuevos desequilibrios llevarán al reequilibrio que se constituirá caracterizando el próximo nivel. Sin embargo, una serie de nuevos cuestionamientos es levantada por el sujeto y puede llevar a una falsa impresión de regresión cuando el mismo no domine tales conceptos.

En 'periodo de las operaciones formales', que inicia alrededor de los 11-12 años, el sujeto pasa a operar a partir de hipótesis y no apenas sobre los objetos. Según Piaget (1983), "el conocimiento ultrapasa la propia realidad para ser parte de lo posible y para relacionar directamente lo posible a lo necesario" (p. 27). Por tanto, el sujeto necesita realizar una operación deductiva que va de las hipótesis a sus propias conclusiones, o sea, una operación que se efectúa sobre operaciones o de la relación que se establece sobre relaciones. Operando en este nivel de inteligencia, el sujeto pasa a demostrar un tipo más complejo de reversibilidad donde se observan de manera concurrente la inversión, la reciprocidad, la previsibilidad, acción y reacción.

Según Eichler (2015), incluso con el vasto trabajo y la inmensa propagación, la teoría piagetiana continúa encontrando interpretaciones di- 
versas. Ya citamos anteriormente la reafirmación de Lourenço (2016) para la creencia de Piaget en la continuidad del desarrollo cognitivo ya que las estructuras formales pueden ser infinitamente elaboradas. Aun así, se ven trabajos recientes, como el de Subía y Gordón (2014), apuntando que con las operaciones formales "se espera contar con una estructuración cognitiva definitiva; la cual se conserva, durante el resto de la vida adulta" (p. 75).

Otro ejemplo, ahora sobre la 'visión recibida' de Piaget discutida por Lourenço (2016) es vista en el artículo de Naranjo y Peña (2016) en que pretenden discutir el pensamiento lógico-abstracto, más lo hacen sin utilizar ni una citación directa a Piaget. Este trabajo aborda diferentes teorías cognitivas y discurre brevemente sobre la 'teoría psicogenética de Piaget', más sin, de hecho, citar la única obra de Piaget encontrada en sus referencias.

Comprender verdaderamente la visión piagetiana implica en el estudio directo de su obra. Existe una gran complejidad involucrada en la búsqueda, estructuración y caracterización de la evolución del desarrollo cognitivo humano. Una de las formas de comprender la estructuración formulada por Piaget para explicar los períodos de desarrollo y comprender que ellos se constituyen como procesos sucesivos de equilibrio. Piaget (1956) afirma que:

A partir del momento en que el equilibrio es alcanzado sobre un punto, la estructura es integrada en un nuevo sistema de formación, hasta un nuevo equilibrio siempre más estable y con campo siempre más amplio. Conviene recordar que el equilibrio se define por la reversibilidad. Decir que hay camino para el equilibrio significa que el desarrollo intelectual se caracteriza por una reversibilidad creciente. La reversibilidad es la característica más aparente del acto de inteligencia, que es capaz de desvíos y de retornos (p. 42).

Piaget presenta los procesos de equilibrio mientras desvela el desarrollo cognitivo, y para eso hace un abordaje de los procesos y estructuras necesarias para la consolidación de estos procesos de un punto de vista multifocal, pero destacando su abordaje a los factores psicológicos y fisiológicos involucrados. Esta integración de los sistemas psicológico y fisiológico quedó conocida como el paralelismo psico-fisiológico de Jean Piaget.

\section{El paralelismo psico-fisiológico de Jean Piaget}

Durante el gran periodo de producción científica de Jean Piaget no existían la tecnología de diagnóstico e imagen (RMI, EEG, ERP y otros) 
o los actuales datos sobre el funcionamiento y el comportamiento del cerebro al que hoy tenemos acceso. Según Arsalidou y Pascual-Leone (2016), Piaget desarrolló su teoría sin los beneficios de la neurociencia actual. Aun así, el conocimiento biológico y los estudios sobre adaptación y evolución conducidos por él ya apuntaban hacia la integración de los sistemas psicológico e fisiológico. Corso (2009) utiliza las proposiciones de Antonio M. Battro para afirmar que Piaget estudió el cerebro sin la neurología y que ahora es el momento de interpretar los resultados que él obtuvo con base en la neurología actual. El propio Battro (1996) hizo importantes afirmaciones para demonstrar la preocupación fundamental de Piaget, como un 'psicólogo de la inteligencia', con los procesos generales del equilibrio orgánica encima de los procesos particulares del sistema nervioso. Según Battro (1996):

Piaget tenía una conciencia muy clara de los límites de sus competencias científicas en este campo - en el célebre debate con Chomsky afirmó: "no sé nada de neurología" (Piatelli-Palmarini, 1979, p. 290) - sin embargo, intuía con razón, que las neurociencias de su época poco podían contribuir para el estudio del desarrollo de la inteligencia, tal como él la concebía (p. 2).

Piaget recalcó en todo momento la influencia de los factores biológicos en el desarrollo de la inteligencia y para la adquisición de conocimiento. Estos factores estarían conectados al que llamó de sistema epigenético, demostrando interacciones entre genoma y medio físico durante el desarrollo de esos procesos que se manifestarían por la maduración del sistema nervioso. El paralelismo psicofisiológico dispuesto por él acompañó el desarrollo de su teoría y puede ser visto en diversos trabajos, aunque en algunos momentos sea destacable el papel poco conocido del comportamiento neuronal en la época. Battro (1996) reafirma la defensa de Piaget del paralelismo psicofisiológico cuando cita el Tratado de Psicología Experimental, él afirma:

Podemos recordar que Piaget siempre defendió el llamado "paralelismo psicofisiológico". Fue muy explícito al respecto: "si el paralelismo entre los hechos de la conciencia y los procesos fisiológicos responden a un isomorfismo entre los sistemas verdaderamente implicados y los sistemas materiales de orden causal, es evidente entonces que ese paralelismo conduce igualmente no solo a una complementariedad más, a fin de cuentas, a una esperanza fundada del isomorfismo entre los esquemas organicistas y los esquemas lógico-matemáticos utilizados por los modelos abstractos" (p. 2). 
Para Piaget (1973) esos 'factores psico biológicos' no son las únicas atenuantes. Analizando diferencias observadas en el desarrollo de sujetos pertenecientes a diferentes grupos sociales, diferentes culturas, e incluso en sujetos de realidades semejantes, él presenta diversos ejemplos donde se observan atrasos o anticipaciones en el desarrollo (decalagens), y deja claro que otros factores más allá de la maduración biológica están involucrados en esos procesos. En este contexto, Piaget (1973) afirma:

...que se trate de acciones ejecutadas individualmente o de acciones practicadas en común con permutas, colaboraciones, oposiciones, etc, se encontrarían las mismas leyes de coordinación y de regulación que llevarían las mismas estructuras finales de operaciones o de cooperaciones, mientras cooperaciones; así se podrían considerar la lógica, mientras forma final de los equilibrios, como siendo simultáneamente individual y social, individual cuan general o común a todos los individuos y también social, cuan general o común a todas las sociedades (p. 57).

$\mathrm{Al}$ abordar las relaciones que se establecen entre las funciones cognitivas y los factores sociales, Piaget (1973) asume ser indispensable "comenzar oponiendo las "coordinaciones generales" de las acciones colectivas a las transmisiones culturales particulares que se cristalizaron de manera diferente en cada sociedad" (p. 56). Explicando el paralelismo psicofisiológico, Eichler y Fagundes (2005) asumen el comportamiento como resultado de procesos neurónicos que pueden ser provocados por estímulos externos y adhieren a las ideas de Bunge y Ardila (1987), postulando que:

...el desarrollo humano sería comprendido como un proceso bio social de reorganización neural entrelazado con socialización. Así, solamente una bio psicología desarrollista unida con una psicología social incluiría la promesa de explicaciones científicas que viesen al desarrollo (p. 259).

Muchas preguntas impregnan el debate sobre los papeles psicológico y fisiológico de las estructuras de pensamiento, de la adquisición de conocimiento, del funcionamiento del cerebro y de la mente. Inicialmente, distinguir los papeles del cerebro y de la mente ya trae controversias. Mario Bunge (1988), en el prefacio de su obra El problema mente-cerebro: un enfoque psicobiológico, destaca que en el inicio del siglo $\mathrm{XX}$ algunos eminentes psicólogos y neurofisiólogos diseminaban la idea de que hablar sobre la mente no era científico, más si una superstición que debería ser abandonada. También se deberían abandonar conceptos teológicos sobre el alma, así como ideas de capacidad, disposición, estado y proceso mental. La ciencia apenas aceptaría tratar de cosas concretas. Para Bunge 
(1988), había la necesidad de transformar la concepción de que la mente es un conjunto de actividades cerebrales:

La neurofisiología es necesaria, mas no lo suficiente, pues tiende a descartar categorías psicológicas, como la de propósito y la de pensamiento. Y la psicología, que, aunque sea igualmente necesaria, tampoco es suficiente -a menos que sea fisiológica- una vez que tiende a olvidar del sistema nervioso (p. 16).

¿De qué maneras la epistemología genética y las neurociencias cognitivas definen el papel del cerebro y de la mente? Las Neurociencias están enfocadas hacia el estudio del cerebro, de las redes neuronales, de las células y de sus componentes genéticos. Visto así, hay quien diría que Piaget se habría apenas preocupado con la psicología, con el estudio del alma, del espíritu. Más, obviamente, no fue apenas eso. Su intento de construir una explicación biológica para el conocimiento trazó un camino paralelo para el estudio del espíritu. Al analizar estos puntos, Corso (2009) afirma que la:

...estructuración del sistema nervioso aparecería como intermediaria entre la fisiológica y la mental, ya que la reacción nerviosa garantizaría la transición entre la asimilación fisiológica y el funcionamiento del organismo y la asimilación cognitiva o interacción de objetos o situaciones en los esquemas de acción (p. 228).

El punto clave del debate sobre el problema mente-cerebro, discutido desde Platón hasta los días actuales se resume en: quién es que percibe, siente, recuerda, imagina, ¿desea y piensa? Es la mente/alma/espíritu o es el cerebro? Por lo menos dos líneas de pensamiento estarán expresadas aquí. Una que defiende la mente como propietaria de todos los estados y procesos mentales y la otra que da al cerebro el destaque, pues la mente no es independiente de él y, por lo tanto, la propia mente es un conjunto de funciones o actividades cerebrales. Correa, Agila, Pulamarín y Palacios (2012) citan a Javier Monserrat para afirmar que la mente funciona como un conjunto interconectado de "recursos físicos, biológico-neurológicos y psíquicos que sustentan el organismo humano vivo los procesos de detección de información y elaboración de las respuestas adaptativas al medio" (p. 129). Además, ellos también apuntan una "integridad manifiesta entre el cuerpo y el cerebro" (p. 130).

Por las varias demostraciones dadas por Piaget de que la mente funciona de modo interconectado al cerebro, podemos creer que él compone el grupo llamado por Bunge (1988) de dualistas psicofísicos. Bunge 
también analiza que existen dos variedades más populares de dualismo psiconeural: el paralelismo y el interaccionismo, ambas empíricamente equivalentes. Para él, los interaccionistas, como René Descartes (15961650), Karl Raimund Popper (1902-1994), Wilder Penfield (1891-1976) y aquí se añade Jean Piaget (1896-1980), conciben el cerebro (la parte física) y la mente (la parte mental) como interactuando mutuamente. El cerebro sería para ellos la base de la mente, aunque sea controlado por ella. Según Piaget (1973):

...los factores psico biológicos están lejos de ser los únicos actuantes. Si, con efecto, no interviniese si no una acción continua de la maduración interna del organismo y del sistema nervioso, las fases no serían apenas secuenciales, mas también conectados a fechas cronológicas, relativamente constantes, como lo son la coordinación de la visión y de la sujeción, alrededor de los 4-5 meses, la apariencia de la pubertad, etc. Conforme los individuos y los medios familiares, escolares o sociales en general, se encuentran, entre niños de una misma ciudad, progresos o atrasos muchas veces considerables, que no contradicen la orden de sucesión, que permanece constante, pero que muestran que a los mecanismos epigenéticos se añaden otros factores (p. 54).

Habiendo traído a esta discusión relevantes cuestiones, principalmente abordando diversos puntos interrelacionados con las neurociencias, es importante analizar las contribuciones de investigaciones de este campo para el tema en debate. Battro (1996) afirma que "es difícil, con efecto, encontrar una sola citación de Piaget en los textos de neurociencias cognitivas más difundidos e influyentes de la actualidad" (p. 2). A pesar de eso el autor sugiere que:

... el modelo de las operaciones mentales construido por Piaget a lo largo de toda su vida es uno de los más aptos para estudiar los caminos neuronales cognitivos (neurocognitive paths). Hoy llegó el momento de hacerlo con rigor y precisión. Las neurociencias de estos últimos años progresaron de tal manera que nos permiten avanzar decididamente en el campo cognitivo, algo que era impracticable en la época de Piaget (p. 3).

Buscando establecer aproximaciones posibles entre la epistemología genética de Piaget y las neurociencias cognitivas, en este artículo se escogió rescatar los trabajos del biofísico americano Herman T. Epstein, que serán resumidos en la próxima sección. 


\section{Las etapas cerebrales de Herman T. Epstein}

Un número significativo de trabajos utilizando tecnologías no invasivas para la construcción de imágenes y el estudio de la fisiología cerebral, principalmente mientras actividades son realizadas por el sujeto en estudio, ha traído mayor entendimiento sobre el funcionamiento del cerebro $y$, más que eso, reafirman la teoría piagetiana y demuestran la construcción de la mente.

La evolución de tecnologías y de conocimiento sobre las estructuras que componen el cerebro y su funcionamiento permitirán que antiguas creencias como las de que la maduración cerebral ocurría precozmente, de que la pérdida de neuronas era irreversible y de que la anatomía cerebral no podría ser alterada con la experiencia, empezaron a ser cuestionadas mientras la noción de plasticidad comenzó a ser construida. A partir de ahí, el creciente número de estudios realizado fue construyendo nuevos contornos para el conocimiento científico sobre el funcionamiento del cerebro y de la mente.

Epstein realizó un extenso trabajo sobre el desarrollo cerebral (1974; 1990; 1999), en algunos de ellos acompañando el aumento de peso de ese órgano y aumento de la circunferencia del cráneo en niños y adolescentes. Él es uno de los investigadores ligados al campo neurocientífico que proponen compromisos de su trabajo como el de Piaget. En su trabajo intitulado The roles of brain in human cognitive development (1999), Epstein abre el artículo enfatizando la relación entre desarrollo cerebral y la descripción piagetiana de las etapas del desarrollo. Según él:

El desarrollo cerebral en humanos ocurre en etapas establecidas en correlación con el principio de etapas de Piaget para el desarrollo del pensamiento. Esto provee una descripción del desarrollo cognitivo como resultado parcial, a partir de, y dependiente de eventos biológicos ocurridos en el cerebro. Evidencias muestran que algunas estructuras cerebrales eventuales dependen de una combinación de eventos biológicos instructivos o datos experimentales (p. 1).

Battro (1996) afirma que Piaget siempre defendió la idea de un proceso general de equilibrios y desequilibrios cognitivos que prolongan las regulaciones y desregulaciones orgánicas. Ya Corso (2014) destaca el hecho de que para Piaget el comportamiento esté expuesto a todos los desequilibrios por ser dependiente de un medio que es ilimitado e inestable. Así, ocurre la creación de un sistema permanente de compensaciones para las perturbaciones externas y de ajustes al mismo tiempo retroactivo y anticipador. Piaget (1973) dice: 
El desarrollo individual es, en verdad, función de actividades múltiples en sus aspectos de ejercicio, de experiencia o de acción sobre el medio, etc. Interviene, sin cesar, entre las acciones de las coordinaciones particulares o cada vez más generales. Esa coordinación general de las acciones supone entonces sistemas múltiplos de auto-regulación o equilibración, que dependerán de las circunstancias, tanto cuanto las potencialidades epigenéticas. Las propias operaciones de la inteligencia pueden ser consideradas formas superiores de esas regulaciones, lo que muestra, al mismo tiempo, la importancia del factor equilibración y su relativa independencia, en lo que respecta a las pre-informaciones biológicas (pp. 54-55).

La intención de Epstein (1974) fue demostrar que el cerebro humano posee períodos de gran crecimiento en peso, y que estos períodos 132 no están directamente relacionados al crecimiento general del cuerpo. Utilizando datos de búsquedas anteriores realizadas por Coppoletta y Wolbach, Reed y Stuart, Blinkov y Glezer (en Epstein, 1974), él encontró en ese análisis sobre el aumento del peso cerebral que los períodos de especial crecimiento de órganos (corazón, pulmones, hígado y riñones) y del cuerpo como un todo, generalmente no coincidían con los períodos característicos de crecimiento cerebral. Para definir esos períodos de crecimiento rápido, del cerebro y de la mente, él acuño el termino Frenoblenoses, del griego phreno -cerebro o mente, blysis- surgimiento, crecimiento de materia y nósis-conocimiento.

Epstein organizó su cartografía cerebral en períodos que intercalan crecimiento rápido y lento. Durante el crecimiento rápido hay un aumento de las células cerebrales (hasta cierto punto) y el esparcimiento de las redes neuronales y sus asociaciones a través de conexiones y el establecimiento de sinapsis. En los períodos de crecimiento lento, estas redes son perfeccionadas, fortalecidas, recortadas. La calidad con que las redes se desarrollan se manifestará en la próxima etapa y servirán como base para el desarrollo de las habilidades siguientes. Estos hechos refuerzan la tesis piagetiana de la construcción del conocimiento, en que la acción del individuo sobre los objetos y el miedo y las respuestas que él recibe al hacer estas interacciones son la base de las asimilaciones necesarias para el avance de sus aprendizajes.

Para cada período de crecimiento rápido identificado por Epstein (1999), corresponde otro, más lento donde él observó que "Durante períodos de crecimiento cerebral rápido el peso cerebral aumenta en media de 5 a $10 \%$ mientras que durante períodos interinos de bajo crecimiento cerebral, el aumento es de tal vez 1\%” (p. 1). Al analizarse los períodos 
descritos y las edades en torno de las cuales se espera el establecimiento de los mismos, se ve una relación íntima que reafirma los estudios de Piaget, como se ve cuando Epstein (1999) dice:

Mientras el niño crece, esos controles de movimientos mejoran con la práctica y la experiencia entonces las redes controlando estos movimientos deben estar siendo pulidas y afiladas y, por tanto, ellas trabajan más eficientemente. Si el niño no gana la experiencia suficiente y de amplia variedad para activar un espectro entero de movimientos, algunas de estas redes de movimiento pueden ser menos que optimas (p. 3).

Al presentar sus evidencias, Epstein deja clara la necesidad de la experiencia (o sea, de la vivencia, experimentación, tentativa/error, en fin, de las asimilaciones) y de la relación con los objetos y el medio para que las estructuras previas sean establecidas con calidad. Sus percepciones acerca del desarrollo cognitivo humano refuerzan la teoría del desarrollo cognitivo en etapas creadas por Piaget. Aún según el autor (Epstein, 1999):

...la evolución ha procedido por el aumento del número de contactos entre áreas (y sus funciones características); esto indica una significativa arborización adicional. Entonces las fases (3-10 meses y 2-4, 6-8, 10-12, y 14-16 años) son ahora hechos experimentales y no apenas teorías. Este aumento repentino del crecimiento cerebral ha sido encontrado ocurriendo desde el inicio más precoz de las etapas del desarrollo del pensamiento de Piaget (Epstein, 1980, 1986; Hudspeth y Pribram, 1990); por lo tanto, ellos son probablemente las bases biológicas de las etapas de Piaget (p. 1).

A fin de demostrar su hipótesis para la aparición de frenoblenosis, Epstein (1974) publicó dos estudios en que dividió esta investigación. En primer lugar, utilizando propiedades biofísicas (medidas de circunferencia de la cabeza y estimativas de peso cerebral) en diversas edades para determinar estos períodos de crecimiento. En seguida, el examen de datos comportamentales que muestren cambios abruptos que pudiesen ser relacionados a los cambios biofísicos cerebrales y que indicasen posibles edades en que buscar los períodos de crecimiento rápido. De esta manera, sus descubrimientos posibilitaran estos esquemas de clasificación que mucho se aproximan de aquellos disponibles por Piaget.

La investigación de Epstein presentó evidencias de la existencia de períodos de rápido crecimiento en peso cerebral y circunferencia del cráneo y aún correlacionó el crecimiento físico del cerebro con el crecimiento funcional de la mente. Según Epstein (1974): 
El espectro de edades para frenoblenosis en humanos puede estar correlacionado con el crecimiento de las habilidades mentales (...) conversando con profesores, tenemos razones para creer que los períodos están relacionados con el crecimiento en las habilidades de aprendizaje. El crecimiento entre 14-15 años está correlacionado con la etapa piagetiana de las operaciones formales, que es generalmente supuesto iniciar después de los 12 años. El crecimiento de los 11 años está correlacionado con el rápido crecimiento de la conceptualización sobre los objetos concretos en el ambiente, que es utilizado en las escuelas para construir, por ejemplo, ideas sobre fracciones y objetos geométricos. El crecimiento de los 7 años coincide con el inicio del aprendizaje formal normalmente asociado con la adquisición de las habilidades de lectura y escritura para la media de los niños (p. 214).

En un estudio que amplio y corroboró la investigación sobre la frenoblenosis, Hudspeth y Pribram (1990) utilizaron cuantificación computarizada de los cambios en el espectro de frecuencia del EEG (QEEG) para establecer relaciones estadísticas entre los estados de regiones cerebrales y maduración. Según ellos, ese análisis brindó evidencias de cinco etapas estadísticamente importantes de la maduración en el QEEG, brindando datos empíricos suficientes para demostrar los períodos de crecimiento rápido y lento sugeridos por Piaget y Epstein. Según ellos, "es razonable concluir que la maduración cerebral ocurre en etapas" (Hudspeth y Pribram, 1990, p. 883).

Así como Piaget, Epstein (1999) presentó que los bebés nacen con "algunas redes neuronales genéticamente establecidas" (p. 2), siendo estas redes necesarias para actividades reflejas como respirar, chupar, detección sensorial, metabolismo, etc. A partir del nacimiento, a través de las interacciones realizadas por el niño (absorción de actos para Epstein, asimilaciones para Piaget) estas redes serán fortalecidas, debilitadas, modificadas, tendrán adicciones, etc. El primer gran período de crecimiento cerebral fue identificado por Epstein (1999) que ocurre entre los 3 y 10 meses. Principalmente, ese crecimiento trabaja para madurar el cerebelo, "facilitando su papel en la activación y control de las acciones motoras" (p 2). En seguida, se inicia un período de bajo crecimiento cerebral que va de los 10 meses a los 2 años donde el niño "practica enteramente complementos de acciones y controles, así mejorando, consolidando y perfeccionándolos en la medida en que las redes son óptimamente dispuestas para este propósito" (p. 3).

Para Epstein (1999), la segunda gran fase de crecimiento cerebral se establece entre los 2 y 4 años, siendo este enfocado para la maduración de los sentidos. Él menciona el desarrollo interconectado del tacto, de la 
visión y de la audición y comenta que "el niño puede ver, oír, saborear, tocar y oler virtualmente al nivel de un adulto al fin de esta fase" (p. 3). En seguida, se sigue un período de bajo crecimiento cerebral que se extiende de los 4 a los 6 años, siendo caracterizada por la "ganancia de experiencia y pericia en la utilización de las redes existentes y de las recién modificadas para realizar actividades sensoriales y motoras" (p. 4), lo que acaba por consolidar estas funciones. Según Epstein (1999):

...diferencias individuales aparecen debido a diferencias en las experiencias de vida a partir de ese punto. Del punto de vista de algo como las fases piagetianas, nuevas funciones van siendo adquiridas y afiladas en un cronograma un tanto sin sincronía y entonces vemos el aparecimiento de aquello que llamamos de compensaciones: el esparcimiento de fases de desarrollo encima de las edades (compensaciones verticales) y encima de los dominios paralelos en cada una de las mismas funciones puede ser empleada (compensaciones horizontales) (p. 4).

En este momento, es importante destacar más una vez que las edades en sí no son factores primordiales. El hecho es que la secuencia de las fases se mantiene fija y todos los individuos recorren todos ellos, más fueron identificados en diferentes pueblos y culturas, atrasos o adelantamientos (compensaciones) en relación a las edades en que se manifestaban, de acuerdo con el local donde los testeos eran realizados. Además de los factores biológicos, los factores sociales, de transmisión educativa y cultural fueron destacados por Piaget (1973):

Para que se puedan invocar con certeza los factores biológicos de la maduración, sería preciso estar en condiciones de verificar la existencia, no apenas de una orden secuencial de los estadios, más aún de ciertas fechas medias, cronológicamente fijas, de apariencia; más los resultados de Mohseni muestran, al contrario, un atraso sistemático de los niños campesinos, en relación a los niños de las ciudades, lo que indica, naturalmente, que intervienen otros factores, además de los de la maduración (p. 60).

Hablando sobre el crecimiento de la red neuronal y como esto posibilita la asociación de funciones sensorio-motoras y mentales que antes estaban separadas, permitiendo operaciones más complejas alrededor de los seis años, él destaca que esta adquisición no depende más de un aumento de células nerviosas, más que por medio de estímulos, nuevas redes de comunicación neuronal se establecen y se manifiestan entre redes previamente existentes, lo que para él tenía gran sentido biológico ya que "las únicas redes creadas después de este tiempo serán aquellas actividades con operaciones sensorio-motoras" (p. 5). Epstein (1999), que sigue afirmando: 
Es extremamente importante apuntar que estas novas funciones no son genéticamente programadas porque, si ellas fuesen, virtualmente todos los niños manifestarían estas funciones del pensamiento concreto gravemente con las mismas edades, como hacen durante las primeras etapas de crecimiento cuando virtualmente todos los niños manifiestan funciones sensoriales y motoras típicas. Esto significa que el funcionamiento de esta red aumentada es dependiente de modificaciones por la combinación de entradas hechas por la experiencia e instrucción. ¡Sus funciones deben ser aprendidas! (p.5).

Siguiendo el relato de Epstein, él afirma que encima de esta fase de desarrollo el pensamiento parece ser "casi que enteramente asociativo” (p. 5), y destaca que entre los 8 y 10 años sigue un nuevo período de crecimiento cerebral lento donde ocurre la consolidación de nuevas funciones. Explicando esta cuestión:

Es muy importante notar que la síntesis de nuevas células cerebrales llega a un impase virtual alrededor de la edad de 4-5 años (Winick, 1968). A causa de las limitaciones de sensibilidad en las mediciones, no es posible declarar que ninguna síntesis ocurre después de eso. Más, tan lejos como pueda ser determinado, hay un cesamiento de la actividad de la enzima involucrada en la re-aplicación del DNA (DNA polimerasa) es una asíntota en el DNA total por el cerebro entonces no hay aumento significativo del número de células cerebrales.

La importancia de este hallazgo es esta, porque el cerebro aumenta cerca de $30 \%$ en peso después de esta edad, el peso adicional tiene que ser en aumento de peso por célula cerebral. Mucho del aumento de peso está en el aumento de la arborización de las neuronas, esto significa que elles envían axones y dendritas más alargados y más ramificados para crear conexiones funcionales entre grupos de neuronas localizadas más distantes (Conel, 1939-63; Rabinowicz, 1979). Este aumento en la complejidad de la red se vuelve posible e inevitable al funcionamiento mental más complejo (p. 4).

Alrededor de los diez años fue identificado un nuevo período de rápido crecimiento cerebral, con la identificación de un aumento significativo de la arborización neuronal donde ocurren nuevos contactos y asociaciones con redes pre-existentes. Estos nuevos contactos posibilitan la asociación de funciones del pensamiento concreto, y a partir de ahí existe la posibilidad de la manifestación del pensamiento formal. Aún, más una fase de crecimiento rápido fue identificada entre los 14-16 años, donde la arborización adicional permite conectar funciones del pensamiento concreto que aún no estaban conectadas permitiendo la adición 
de funciones del pensamiento formal que serán sumadas, agregadas en este período. Considerando la visión general de su trabajo, Epstein (1999) concluye que "los esquemas de pensamiento piagetianos han sido confirmados y reconfirmados muchas veces" (p. 7).

\section{Conclusiones}

No hay duda de que el desarrollo cognitivo humano está estructurado en los pilares psicológico y biológico, y que estos pilares están ligados a factores sociales, culturales, educativos y genéticos. Toda la interacción del individuo con el medio que lo envuelve está mediada por estos factores, y son ellos los que dan la base utilizada en las asimilaciones realizadas y posteriores alojamientos. Las diferencias encontradas en las diversas replicaciones de las pruebas piagetianas alrededor del mundo, apenas demuestran la interferencia de estos factores.

En el ámbito de las neurociencias, las etapas del desarrollo humano establecidas por Piaget fueron confirmadas por la extensa investigación de Epstein $(1974,1990,1999)$, que estableció la correlación entre el desarrollo cerebral y las etapas de Piaget para el desarrollo del pensamiento, y que más tarde fue corroborada por Hudspeth y Pribram (1990), Hansen y Monk (2002), Quartz y Sejnowski (1997) entre otros. Siendo así, se demostró que es posible establecer relaciones entre las neurociencias cognitivas y la epistemología genética y no solo eso de que la base de sustentación del polo constructivista en las neurociencias es bastante sólida.

Para además de las etapas del desarrollo, las evidencias demostradas del análisis superficial de los modelos piagetianos, de la adopción de parcialidad y de la omisión de datos, de la no consideración de los trabajos de Piaget como un todo, del apego a obras aisladas en determinados períodos, lecturas secundarias y terciarias de la obra piagetiana y de los problemas de lectura y traducción de los textos originales, etc.; se constituyen en justificaciones suficientes para la poca o casi inexistente divulgación del abordaje constructivista en las investigaciones neurocientíficas.

De este modo, se demostró en este artículo que al contrario de ser un campo homogéneo, infalible, finalizado, o sea, singular, las neurociencias se constituyen de forma plural y heterogénea, apoyadas en sesgos diversos y muchas veces contradictorios, y que los datos empíricos traídos a la discusión permiten que el debate entre los polos innatista $\mathrm{x}$ constructivista no sea cerrado, más se mantenga encendido y, esperamos, en debate propicio. 


\section{Bibliografía}

ARANHA, Gláucio \& SHOLL-FRANCO, Alfred

2012 Caminhos da Neuroeducação. Rio de Janeiro: Ciências e Cognição.

ARSALIDOU, Marie \& PASCUAL-LEONE, Juan

2016 Constructivist developmental theory is needed in developmental neuroscience. Science of Learning, 1, 1-9. https://doi.org/10.1038/npjscilearn. 2016.16

BATTRO, António Maria

1996 Jean Piaget y la neuroeducación. Disponível em https://goo.gl/qduYir [1111- 2018].

BECKER, Fernando

2003 A origem do conhecimento e a aprendizagem escolar. Porto Alegre: Artmed.

BEE, Helen \& BOYD, Denise

2011 A criança em desenvolvimento. Porto Alegre: Artmed.

BUNGE, Mario \& ARDILA, Rubén

1987 Philosophy of psychology. New York: Springer-Verlag.

BUNGE, Mario

1988 El problema mente-cerebro: Un enfoque psicobiológico. Madrid: Editorial Tecnos.

CAREY, Susan, ZAITCHIK, Deborah \& BASCANDZIEV, Igor

2015 Theories of development: In dialog with Piaget. Developmental Review, 38, 36-54.

CORSO, Helena Vellinho

2009 Funções Cognitivas-convergências entre neurociências e epistemologia genética. Educação \& Realidade, 34(3), 225-246, Set/Dez.

2014 Cérebro e mente: convergências entre os modelos de Piaget e Fuster. Schème: Revista eletrônica de psicologia e epistemologia genéticas, 6, nº especial.

DONGO-MONTOYA, Adrian Oscar

2013 Resposta de Piaget a Vygotsky: convergências e divergências teóricas. Educação \& Realidade, 38(1), 271-292. Porto Alegre.

EICHLER, Marcelo Leandro

2015 Acerca das citações à obra de Jean Piaget em revistas indexadas. Schème: Revista Eletrônica de Psicologia e Epistemologia Genéticas, 7, 35-57.

EICHLER, Marcelo Leandro \& FAGUNDES, Léa

2005 Atualizando o debate entre Piaget e Chomsky em uma perspectiva neurobiológica. Psicologia: Reflexão e Crítica, 18(2), 255-266.

EPSTEIN, Herman T.

1974a Phrenoblysis: Special Brain and Mind Growth Periods. I. Human Brain and Skull Development. Developmental Psychobiology, 7(3), 207-216.Waltham, Massachusetts.

1974b Phrenoblysis: Special Brain and Mind Growth Periods. II. Human Mental Development. Developmental Psychobiology, Waltham, Massachusetts, 7(3), 217-224.

1990 Stages in human mental growth. Journal of Educational Psychology, 82(4), 876-880. 
1999 The roles of brain in human cognitive development. Disponível em http:// www.brainstages.net/stages [11-11-2016].

GAZZANIGA, Michael S. \& HEATHERTON, Todd F.

2005 Ciência psicológica: mente, cérebro e comportamento. Porto Alegre: Artmed.

HANSEN, Linda \& MONK, Martin

2002 Brain development, structuring of learning and Science education: where are we now? A review of some recent research. International Journal of Science Education, 24(4), 343-356.

HOUDÉ, Olivier

2009 Dez lições de psicologia e pedagogia: Uma contestação das ideias de Piaget. São Paulo: Ática.

HUDSPETH, William J., \& PRIBRAM, Karl H.

1990 Stages of brain and cognitive maturation. Journal of Educational Psychology, 82(4), 881-884. http://dx.doi.org/10.1037/0022-0663.82.4.881

JARAMILLO, Lilian Mercedes, \& PUGA, Luis Alberto

2016 El pensamiento lógico-abstracto como sustento pra potenciar los procesos cognitivos en la educación. Sophia: colección de Filosofía de la Educación, 21(2), 31-55.

LOURENÇO, Orlando M.

2016 Developmental stages, Piagetian stages in particular: A critical review. New Ideas in Psychology, 40, 123-137.

MACHADO, Diandra Dal Sent

2015 Epistemologia genética e neurociências: construção do sujeito cognoscente. 2015. 93 f. Dissertação (Mestrado em Educação) - Faculdade de Educação, Universidade Federal do Rio Grande do Sul, Porto Alegre.

NIAZ, Mansoor

1998 The epistemological significance of Piaget's developmental stages: a Lakatosian interpretation. New Ideas in Psychology, 16, 47-59.

PAPALIA, Diane E. \& FELDMAN, Ruth Duskin

2013 Desarrollo humano. Porto Alegre: AMGH.

PIAGET, Jean

1956 Les stades du développment intelectuel de l'enfant et de l'adolescent. In P. Osterrieth, J. Piaget, R. De Saussure, J. M. Tanner, H. Wallon, R. Zazzo, A. Rey, Le problème des estades en psychologie de l'enfant; symposium de l'Association de Psychologie Scientifique de Langue Française (pp. 33-42). Paris: Presses Universitaires de France,

1973 Psicologia e epistemologia: Por uma teoria do conhecimento. Rio de Janeiro: Forense Universitária.

1983 A epistemologia genética / Sabedoria e ilusões da filosofia; Problemas de psicologia genética. São Paulo: Abril Cultural.

QUARTZ, Steven R. \& SEJNOWSKI, Terrence J.

1997 The neural basis of cognitive development: a constructivist manifesto. Behavioral and Brain Sciences, 20, 596. Cambridge University Press.

SUBÍA, Andrés \& GORDÓN, Jacqueline

2014 Esbozo crítico sobre las estructuras cognitivas: génesis del pensamiento científico. Sophia: colección de filosofía de la educación, 16(1), 71-82. 
VILATUÑA, Fausto, GUAJALA, Diego, PULAMARÍN, Juan José, \& ORTIZ, Walter

2012 Sensación y percepción en la construcción del conocimiento. Sophia: colección de Filosofía de la Educación, 13(1), 123-149.

Fecha de recepción de documento: 20 de julio de 2018 Fecha de revisión de documento: 25 de agosto de 2018 Fechas de aprobación de documento: 22 de septiembre de 2018 Fecha de publicación de documento: 15 de enero de 2019 